\title{
SUSTAINABILITY OF LONG-TERM TREATMENT IN A RURAL DISTRICT: THE LUSIKISIKI MODEL OF DECENTRALISED HIV/AIDS CARE
}

\author{
Nathan Ford, $M S c$ (Virol) \\ Hermann Reuter, $M B C h B, H E D$ \\ Martha Bedelu, $M D, M P H$ \\ Médecins sans Frontières, Lusikisiki, E Cape \\ Helen Schneider, $M B C h B, M P H, P h D$ \\ Centre for Health Policy, University of the Witwatersrand, Fohannesburg \\ Helmuth Reuter, $M B C h B, F C P, M M e d, F R C P, P h D$ \\ Ukwanda Centre for Rural Health, Stellenbosch University, Tygerberg, W Cape
}

\begin{abstract}
Antiretroviral therapy (ART) is slowly rolling out across South Africa, but coverage is highly variable between and within provinces. The chronic shortage of health care workers is recognised as one of the major bottlenecks to scaling up treatment, ${ }^{1}$ and this has the biggest impact in rural areas where the human resource crisis is most acute. ${ }^{2}$
\end{abstract}

For the past three years Médecins sans Frontières (MSF) has been supporting a programme to provide care and treatment for people with HIV/AIDS in the local service area of Lusikisiki, a subdistrict of 150000 inhabitants in the Eastern Cape serviced by one hospital and 12 clinics. Lusikisiki represents one of the poorest and most densely populated rural areas of South Africa. Less than half the population live in formal housing and up to $80 \%$ live below the poverty line.

With just 5 doctors per 100000 people, Lusikisiki is 14 times below the national average and less than the average for Sierra Leone, DRC and Zimbabwe. ${ }^{3}$ An assessment done by MSF in early 2003 found that electricity was only available in a third of clinics and the supply of electricity was unreliable in half of those; only $8 \%$ had running water or a phone, and half lacked nursing accommodation. Drug supply management was a major problem, with up to 60 Essential Drugs List drugs missing at some clinics. Around half of all nursing posts were (and remain) vacant, and a chronic lack of auxiliary staff meant an increased burden of tasks that further limited direct patient care.

Nevertheless, the implementation of HIV care at the primary care level, through task shifting, community mobilisation, and the use of volunteers, has allowed the rapid scale-up of treatment even in this understaffed and poorly equipped setting. Within 2 weeks of the National Operational Plan for Comprehensive HIV Care being launched in October 2003, the first person was initiated on ART, and in less than 3 years, by October 2006, there were almost 2200 people on antiretrovirals (ARVs), including 110 children.

This paper describes how the integration of HIV care and treatment including ART into primary health care in Lusikisiki managed to overcome the challenges of working in a resource-poor rural area to achieve good coverage and outcomes in a relatively short space of time. ${ }^{4}$

\section{OUTCOMES OF INTEGRATING HIV SERVICES INTO} CLINIC CARE

An overview of HIV/AIDS services is given in Table I, together with some of the main outcome indicators.

The aim from the outset was to demonstrate the feasibility of initiation and rapidly scaling up ARV treatment at the clinic level. A cohort analysis of outcomes for people who have been on treatment for more than 12 months shows satisfactory immunological recovery and viral suppression (Table II).
The greater proximity and acceptability of services at the clinic level has led to a much faster enrolment of people on treatment and much better patient retention. Only $2 \%$ of people are lost to follow-up in the clinics, compared with 19\% at the hospital. This higher drop-out rate at the hospital could be due to sicker people starting treatment (higher early mortality), people having to travel further, less preparation of ARV users, and less effective follow-up of defaulters. Mortality in the hospital (13\%) appears to be lower than in the clinics $(16 \%)$. Mortality and loss to follow-up combined is much higher (32\%). While mortality among those lost to follow-up cannot be known, it is expected to be high. 


\section{TABLE I. OVERVIEW OF HIV SERVICES IN LUSIKISIKI}

\begin{tabular}{lll} 
Services & Outputs \\
\hline
\end{tabular}

VCT

Multiplication of entry points through clinics; comprehensive service by lay counsellors who do counselling and testing; provision of treatment encourages testing

\section{Condom distribution}

Community engagement to support prevention

\section{PMTCT services}

Provision of PMTCT services at all clinics with VCT offered at entry

Introduction of dry blood spot PCR testing

\section{HIV/TB integration}

Fluorescent microscope introduced at the hospital

VCT offered to all TB patients

$A R V$ and TB treatment offered at same clinic

\section{ARV therapy}

Provision of ARV at the clinic level

Nurses initiate and manage treatment
46039 tests done in the last 3 years representing two-thirds of adult population. VCT uptake increased 4-fold from 4874 tests in 2002 to 18809 tests in 2005

110000 condoms distributed monthly through 450 points in the community

Uptake of testing increased from 26\% in 2003 to $89 \%$ of women attending antenatal clinics in 2006

PCR positivity rate of $12 \%$ among last 200 HIV-exposed newborns

Number of tests done each month $(N=1100)$ found to be positive has increased from $15-20 \%$ to $25-30 \%$

$22 \%$ increase in proportion of TB patients with a known HIV status between the last two supervision visits now standing at $70 \%$ tested

See Table II and Fig. 1
TABLE II. TWELVE-MONTH OUTCOMES AT CLINICS AND THE HOSPITAL, LUSIKISIKI

\begin{tabular}{lrr|} 
& Clinics & Hospital \\
\hline No. of patients starting ARVs & 595 & 430 \\
Remaining on treatment & $482(81 \%)$ & $289(67 \%)$ \\
Deaths & $100(17 \%)$ & $58(13 \%)$ \\
Lost to follow-up & $13(2 \%)$ & $83(19 \%)$ \\
CD4 > 200/ $\mu$ l & $87 \%$ & $75 \%$ \\
Viral load undetectable & $90 \%$ & $78 \%$ \\
*All had started ARVs between January 2004 and June 2005 and completed at \\
least 12 months of treatment by July 2006. \\
\hline
\end{tabular}

Overall coverage (treatment as a proportion of need) is impressive: according to established modelling, the programme had achieved 95\% coverage for 2005. (This calculation is derived from the ASSA Model (www.assa.org.za/aidsmodel.asp). It calculates all people entering Stage IV and requiring treatment in 2005; it does not cover the backlog of people needing treatment that would have accumulated in previous years.) At this level of coverage people arriving at the clinics with HIV/AIDS are far less sick than was previously the case. In the inception phases of the programme many people were so sick that they had to be carried to clinics; every day there would be several new arrivals 'by wheelbarrow'. This is rarely seen today. This 'catch up of the backlog' is reflected in the statistics: in early 2004, 50\% of service users at the hospital and 40\% at clinics arrived with a CD4 count < 50 cells/ul; by the end of 2005 this had dropped to $16 \%$ at both hospital and clinics. Because people are arriving with better immune status, clinical management is easier, freeing up the time spent on each person so that more people can be seen.
APPROACHES TO SUPPORT CLINIC-BASED CARE

The approach taken in Lusikisiki is in keeping with the World Health Organization's public health approach to HIV/AIDS services. WHO is promoting a radical departure from traditional models that depend on specialists; instead, task shifting is encouraged to enable nurses to prescribe and dispense ARV therapy and community workers to deliver a wide range of HIV services. The WHO has made it clear that it supports a public health model of service delivery that uses standardised, simplified and decentralised systems to maximise the role of primary health care and community-led care. ${ }^{5}$

The delivery of HIV services in Lusikisiki has depended on three approaches: decentralisation to primary health care to spread the workload across facilities, task shifting within services, and the creation of new human capacity to support the system.

\section{DECENTRALISATION: CLINIC-BASED ARV THERAPY}

The National Plan states that 'where access to a district hospital is limited, ARV initiation may occur at lower level facilities and mobile clinics where the requisite expertise is available.' Experience from other countries has shown that decentralisation of services is a highly effective way of increasing patient enrolment rates without compromising quality of care. ${ }^{7}$

The main rate-limiting step to increasing treatment coverage in Lusikisiki, as elsewhere, is the initiation of therapy. The current practice in many places of 'down referral' - starting people at the hospital, and then passing them on to the clinic - creates a bottleneck in treatment and an unnecessary 
shuttling of service users, prescriptions and laboratory results between institutions. It was with this perspective that the Lusikisiki programme from the outset provided ARVs at the clinic level.

For the first year of the programme enrolment increased at a similar pace at the clinics and hospital, but from then on enrolment at the hospital reached a plateau and then began to decline, suggesting a saturation of services. In contrast, enrolment in the clinics continues to increase (Fig. 1). Enrolment is increasing faster in the clinics because of multiple service points, and services are integrated into general consultations and not dependent on specific staff. The much lower number of people who are lost to follow-up and the faster enrolments at the clinics are a clear indication of service user preference to follow treatment at sites close to where they live. These indicators also point to the more 'user friendly' services that are provided by clinics. While the community initially had more confidence in a doctor, this preference was soon outweighed by the advantages of clinicbased care that is proximal, part of the community and supported strongly by community groups.

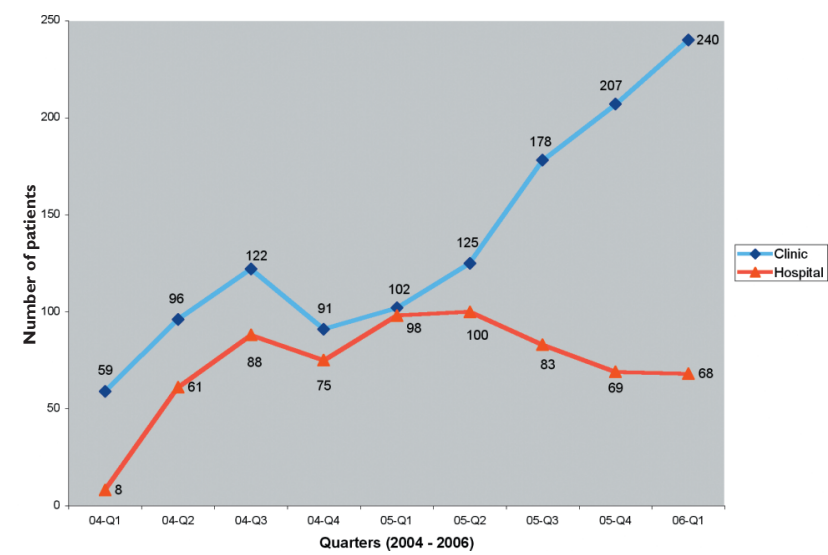

Fig. 1. Enrolment at clinics compared with hospital. (NB: this graph shows the number of patients newly enrolled into treatment for each quarter. Numbers are not cumulative.)

TASK SHIFTING: NECESSARY AND DESIRABLE

The provision of treatment at the clinic level inevitably meant a significant increase in service users within a system that was understaffed and poorly equipped. This was dealt with through a radical task shifting within health staff, the creation of new lower cadre posts, and a strong community engagement to support the health system.

Task shifting recognises the competences of actors across the health service and in this respect it is a worthwhile goal in itself. However, it is also a practical, necessary response to the severe shortage of nurses. Last year, 37\% of nursing posts in the Eastern Cape were vacant. ${ }^{8}$ In the meantime utilisation of services has increased. In Lusikisiki, while overall utilisation of clinic services almost doubled, from 16465 in April 2004 to 28191 in April 2006, the number of professional nurses has not changed (30). This represents a near-doubling of workload that would have been impossible to manage without task shifting. The nurse-patient ratio (number of patients seen per nurse per clinical work day) has increased from 29 in 2003 to 47 in 2006; this compares with a national average of $29.4^{\circ}$

Through training, mentoring and supervision, the running of the ARV programme was delegated to primary health care nurses and community health workers. Table III outlines the roles of the various team members in the Lusikisiki programme compared with traditional functions. Task shifting was a departure from a model that relies heavily on the most sparse human resources in the system by shifting as much responsibility as possible to lower cadres while providing professional oversight for quality control.

All clinics receive regular doctor support via a mobile visit to support the overall service. Nurses receive extensive preparation in HIV management, including staging and initiation of ARV therapy in uncomplicated cases and routine follow-up. In the two larger clinics they are supported by pharmacists' assistants for drug management.

\section{NEW HUMAN CAPACITY}

\section{Adherence counsellors as patient advocates}

At the centre of the Lusikisiki model are the adherence counsellors - lay workers who receive training through workshops and on-site mentoring and are employed full-time in the Lusikisiki programme. Within a few months they are able to support all the key processes for running a clinic-based HIV service. This includes service user support, treatment preparedness, facilitating support groups, arranging follow-up visits, teaching people on ARVs to package pillboxes, addressing problems in adherence, and collecting and collating statistics. Adherence counsellors work closely with other community actors: volunteer workers (community caregivers), various support groups, adherence and clinic committees and treatment activists (TAC).

Debates on scaling up voluntary counselling and testing (VCT) have focused on whether testing should be voluntary or routinely performed. Often missing from the debate is the basic need to increase capacity (people and space) to do VCT. According to National Department of Health policy, nurses must do testing, but this limits the number of tests that can be done and fragments the VCT process. In Lusikisiki, the rapid uptake of VCT services was only possible because of the increased capacity provided by counsellor-based testing, an approach that has also proven its effectiveness elsewhere. ${ }^{10}$

In contrast to the model of community-based health workers, adherence counsellors are facility-based and support a range of activities related to HIV/AIDS in the clinics, while at the same time advocating rights of service users and participating in decision-making about health services. The low rate $(2 \%)$ of loss to follow-up in clinics can be attributed largely to the work of the adherence counsellors.

While the critical role played by adherence counsellors is recognised by clinic staff and service users, their function is not as yet accommodated by Department of Health staffing structures. In Lusikisiki a community-based organisation 


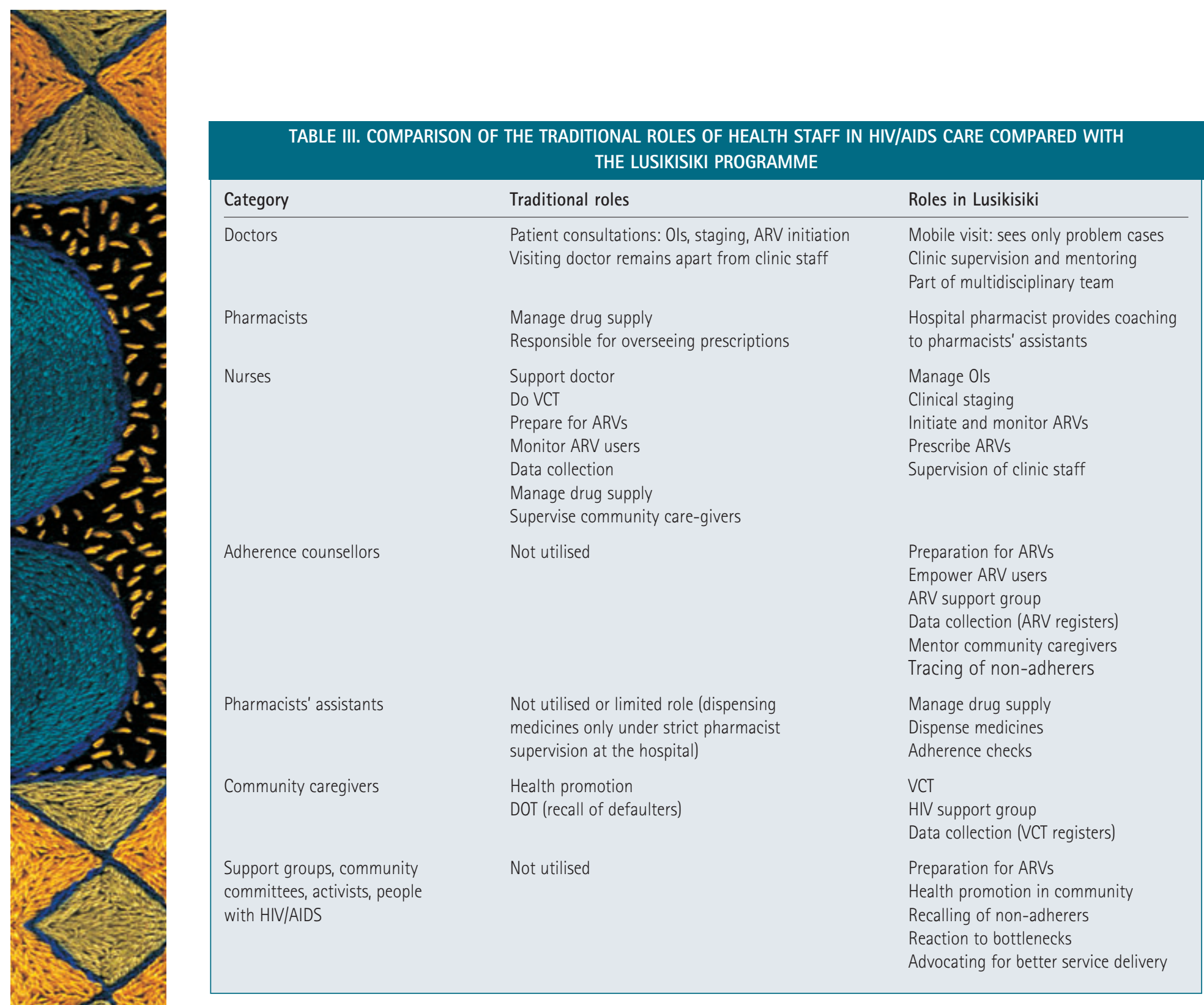

called HAACO (HIV/AIDS Adherence Counsellors Organisation) has recently been formed to ensure that their role is sustained in the long term.

\section{Community support}

Engaging the community in HIV/AIDS care is a proven way to enhance programme quality, in terms of clinical outcomes, adherence rates, and retention. ${ }^{11}$ In Lusikisiki, the community interacts with the HIV services in a number of different ways. General support groups provide peer support for disclosure and testing and do home visits where problems are identified. ARV support groups prepare people for treatment, provide support for adherence and managing side-effects, and seek out and support defaulters. A clinic committee represents service users who feel they have been badly treated, advocates for better infrastructure and drug supply, and monitors HIV programme and condom distribution in the community. An adherence committee made up of community members follows up non-adherers and will take decisions if a clinic team cannot decide on readiness of a person for ARVs. Finally, individual service users make a significant contribution through learning about HIV and sharing their experiences, thereby providing important support to other members of the community.

\section{THE BROADER PERSPECTIVE}

The challenges and solutions described for the Lusikisiki programme are far from unique. Across South Africa health care providers in rural areas are struggling to meet the needs of people with HIV/AIDS in an environment of chronic human resource shortages.

A meeting organised by MSF and the Nelson Mandela Foundation in Maropeng at the end of September 2006 brought together actors from 20 different sites across six provinces to discuss challenges to providing HIV care in rural areas (Eastern Cape, KwaZulu-Natal, Mpumalanga, Limpopo, North West and Free State). ${ }^{12}$ All sites expressed a problem of staff shortages, high staff attrition rates, poor infrastructure and, in many cases, an overwhelming patient load that further encouraged high staff attrition rates. According to one hospital programme manager in the Eastern Cape 'nurses are running away from the programme because of workload and burnout'.

Participants outlined several clear policy barriers preventing scale-up, including an unrealistic interpretation of accreditation requirements, lack of support for nurse 
initiation, and a rigid insistence that nurses do VCT. Those programmes that had been able to reach larger numbers (each providing ARVs for more than 1000 patients) listed among their strengths decentralised initiation of ARVs, community partnerships, task shifting, and good integration with primary health care.

\section{CONCLUSIONS}

The primary health care approach to providing HIV services in Lusikisiki has achieved very high coverage without compromising on quality of care. Integration helped spread the load among all staff, while decentralisation helped to spread the load among different clinics. This was essential given a near-doubling in service users with no increase in nursing staff. Task shifting allowed lay counsellors to test, nurses to initiate ARVs, pharmacists' assistants to manage drug supply, adherence counsellors to support the system and proactively support service users, and community groups to actively engage in service provision. Uptake of testing and treatment is much quicker if these services are offered at clinic level, making more service points that are more readily accessible. Because clinics are part of the local community they are more user-friendly, and so people seek treatment earlier and stay on treatment longer.

The shortage of nurses in rural areas is a priority concern. A number of action points have emerged from the Lusikisiki programme as ways to improve nurse recruitment and retention. These include ensuring an adequate budget for a full complement of clinic staff, recruitment of adequate administrative staff (drivers, clerks, pharmacists' assistants) to ensure that nurses spend their time being nurses rather than being consumed by non-nursing tasks, accreditation and increased remuneration of nurses trained and experienced in HIV, acknowledging the great disparity between non-urban settings by paying maximum rural allowances to staff working in the most challenging rural areas like Lusikisiki, and building and renovating nursing accommodation to meet acceptable standards.

Our experience in Lusikisiki shows that far from being to the detriment of health care services as some have suggested ${ }^{13}$ the provision of ART is having a positive effect on the general quality of primary health care. Improvements in drug supply, diagnostic services, monitoring, staff training, and infrastructural improvements all contribute to improving general primary health care. The strong community ownership of and participation in health care delivery has also had a major benefit in supporting the general quality of health services.

External NGOs with the freedom of flexible budgets and human resources can be very effective in helping establish new models of care. The importance of MSF's role in Lusikisiki was not the provision of human and financial resources, which is a time-limited and unsustainable contribution, but rather the mobilisation of expertise and fostering of partnerships to develop innovative approaches to delivering HIV services.
Following a gradual handover over a period of 18 months, MSF left Lusikisiki in October 2006.

Ensuring sustainability in the face of increasing need will require increased resource inputs from the public sector and full acceptance of the creative approaches to implementation, including task shifting and community involvement. Some of these approaches - such as nurse initiation of treatment - are hampered by a lack of clear policy guidance, while others such as lay counsellor testing - are inconsistent with currrent policy, although in practice they are broadly recognised as the only possible way to respond to the needs. Uncertainty in these and other areas is slowing down the accreditation of sites, with the result that accreditation is preventing rather than enabling treatment rollout. Clear direction at the national level is needed on these critical issues.

While the Lusikisiki programme has performed well up to now, concerted efforts must be made to ensure that rate of enrolment continues to increase in order to prevent the treatment gap from widening. The innovative approaches taken in Lusikisiki and elsewhere have been a response to an overwhelming need for services in the face of poorly staffed and equipped facilities, but it should be recognised that this is the reality in most treatment sites across the country, many of which are reaching saturation point. This model is not just a substitution for suboptimal staff levels in rural areas, but needs to be promoted as a model for best practice everywhere.

\section{Acknowledgements}

We would like to thank Nomalanga Makwedini, Nombulelo Mofokeng, and Thandi Sapepa, from the Eastern Cape Department of Health, for their active support in developing the Lusikisiki programme, and the Nelson Mandela Foundation for financial support. Thanks are also due to Professor Jon Rohde, Department of Public Health, BRAC University, Dhaka, for helpful comments on this paper.

REFERENCES

Van Damme W, Kober K, Laga M. The real challenges for scaling up ART in sub-Saharan Africa. AIDS 2006; 20: 653-656.

2. Padarath A, Chamberlain C, McCoy D, Ntuli A, Rowson M, Loewenson R. Health personnel in Southern Africa: Confronting maldistribution and brain drain. Discussion paper 3. Equinet, Harare, 2003. http://www.equinetafrica.org/ bibl/docs/DIS3hres.pdf (accessed 26 October 2006).

3. United Nations Human Development Report. Country sheet: South Africa. http://hdr.undp.org/statistics/data/countries.cfm?c=ZAF (accessed 26 October 2006).

4. Achieving and sustaining universal access to antiretrovirals in rural areas: the primary health care approach to HIV services in Lusikisiki, Eastern Cape. MSF, Cape Town, October 2006. www.msf.org/sa (accessed 26 October 2006).

Health worker shortages and the response to AIDS. WHO, Geneva, August 2006 http://www.who.int/hiv/pub/advocacy/ttr/en/index.html (accessed 26 October 2006).

6. Operational Plan for Comprehensive HIV and AIDS Care, Management and Treatment for South Africa. Pretoria: National Department of Health, South Africa, 19 November 2003, p. 61.

Ferradini L, Jeannin A, Pinoges L, et al. Scaling up of highly active antiretroviral therapy in a rural district of Malawi: an effectiveness assessment. Lancet 2006; 367: 1335-1342.

8. Public Service Accounts Monitor, Eastern Cape Department of Health Monitoring Brief for 2004/5 Financial Year, p. 7. www.psam.org.za (accessed 26 October 2006).

9. Health Systems Trust. Best available data from 2005. http://www.hst.org.za/ healthstats/149/data (accessed 26 October 2006).

10. Marum E, Taegtmeyer M, Chebet $\mathrm{K}$. Taking scale-up of voluntary HIV counselling and testing in Kenya. JAMA 2006; 295: 859-862.

11. Zachariah $R$, Teck $R$, Buhendwa $L$, et al. How can the community contribute in the fight against HIV/AIDS and tuberculosis? An example from a rural district in Malawi. Trans $R$ Soc Trop Med Hyg 2006; 100: 167-175.

12. www.nelsonmandelafoundation.org (accessed 26 October 2006).

13. McCoy D, Chopra M, Loewenson $R$, et al. Expanding access to antiretroviral therapy in subSaharan Africa: avoiding the pitfalls and dangers, capitalizing on the opportunities. Am J Public Health 2005; 95(1): 18-22. 